\title{
Entanglement in Supramolecular Spin Systems of Two Weakly Coupled Antiferromagnetic Rings (Purple-Cr ${ }_{7} \mathrm{Ni}$ )
}

\author{
A. Candini, ${ }^{1}$ G. Lorusso, ${ }^{1,2}$ F. Troiani, ${ }^{1}$ A. Ghirri, ${ }^{1}$ S. Carretta, ${ }^{1,3}$ P. Santini, ${ }^{1,3}$ G. Amoretti, ${ }^{1,3}$ C. Muryn, ${ }^{4}$ F. Tuna, ${ }^{4}$ \\ G. Timco, ${ }^{4}$ E. J. L. McInnes, ${ }^{4}$ R. E. P. Winpenny, ${ }^{4}$ W. Wernsdorfer,${ }^{5}$ and M. Affronte ${ }^{1,2}$ \\ ${ }^{1}$ National Research Center on nanoStructures and bioSystems at Surfaces (S3), CNR-INFM, 41100 Modena, Italy \\ ${ }^{2}$ Dipartimento di Fisica, Università di Modena e Reggio Emilia, via Campi 213/a, 41100 Modena, Italy \\ ${ }^{3}$ Dipartimento di Fisica, Università di Parma, and Unità CNISM di Parma, via G.P. Usberti 7/a, 43100 Parma, Italy \\ ${ }^{4}$ The Lewis Magnetism Laboratory, School of Chemistry, The University of Manchester, \\ Oxford Road, Manchester M139PL, United Kingdom \\ ${ }^{5}$ Institut L. Néel CNRS, BP166, 25 Av des Martyrs, 38042 Grenoble, France
}

(Received 30 June 2009; revised manuscript received 28 August 2009; published 21 January 2010)

\begin{abstract}
We characterize supramolecular magnetic structures, consisting of two weakly coupled antiferromagnetic rings, by low-temperature specific heat, susceptibility, magnetization and electron paramagnetic resonance measurements. Intra- and inter-ring interactions are modeled through a microscopic spinHamiltonian approach that reproduces all the experimental data quantitatively and legitimates the use of an effective two-qubit picture. Spin entanglement between the rings is experimentally demonstrated through magnetic susceptibility below $50 \mathrm{mK}$ and theoretically quantified by the concurrence.
\end{abstract}

DOI: 10.1103/PhysRevLett.104.037203

PACS numbers: 75.50.Xx, 03.67.- a, 75.40.Cx

Arrays of weakly coupled spins represent an ideal playground for investigating quantum entanglement $[1,2]$. Its controlled generation, however, requires the tailoring of local couplings and the preservation of quantum coherence between mesoscopic systems [3]. These goals are currently being pursued with molecular spin clusters, a rich class of systems that exhibit quantum mechanical features in their magnetic response [4] and allow tailoring local couplings $[5,6]$. This, along with the unmatched variety of the physical properties allowed by chemical synthesis, makes them attractive candidates for applications in molecular spintronics [7] and quantum-information processing [8]. In the latter perspective, antiferromagnetic rings were recently shown to possess a number of key properties for the qubit encoding and manipulation $[9,10]$. These include suitable level structure [11], long decoherence times [12], robustness with respect to functionalization and deposition on metal surfaces [13]. Finally, the capability of engineering weak magnetic couplings between well defined molecular building blocks has been demonstrated [14].

Here, quantum entanglement between two heterometallic spin rings is experimentally demonstrated by lowtemperature susceptibility, and theoretically quantified by deriving the concurrence from a microscopic modeling of the ring dimer. As a preliminary step, we characterize in detail new derivatives of the $\mathrm{Cr}_{7} \mathrm{Ni}$ rings and show that at low temperatures these can be regarded as effective $S=$ $1 / 2$ spins. Antiferromagnetic exchange coupling between two such rings is tailored by supramolecular chemistry and accurately estimated by measuring and simulating specific heat, susceptibility, magnetization and electron paramagnetic resonance (EPR). We find that the inter-ring coupling is small enough to leave unaltered the magnetic properties of each ring, but sufficiently large so as to dominate over long-range dipole-dipole interaction and to induce finitetemperature entanglement in experimentally accessible conditions.

The building block of the supramolecular compounds is the $\left[\mathrm{Cr}_{7} \mathrm{NiF}_{3}(\mathrm{Etglu})\left(\mathrm{O}_{2} \mathrm{Ct}-\mathrm{Bu}\right)_{15}\right.$ (phpy)] ring (in short, purple $-\mathrm{Cr}_{7} \mathrm{Ni}$, after the compound's color), where Etglu $=$ $N$-ethyl- $d$-glucamine and phpy $=4$-phenylpyridine [15]. Here, with respect to the original green- $\mathrm{Cr}_{7} \mathrm{Ni}$ ring derivative [9], five bridging fluorides are substituted by alkoxides [see the inset of Fig. 1(a)]. As shown below, this significantly affects the magnetic levels of each ring. Besides, and most importantly, the presence of a terminal ligand in purple $-\mathrm{Cr}_{7} \mathrm{Ni}$ opens up the possibility of synthesizing novel ring dimers and oligomers [15]. Hereafter we focus on a dimer of such purple derivatives, $\left[\left[\mathrm{Cr}_{7} \mathrm{NiF}_{3}(\right.\right.$ Etglu $)$ $\left.\left(\mathrm{O}_{2} \mathrm{C} t \mathrm{Bu}\right)_{15}\right]_{2}$ (bipy)] (bipy $=4,4^{\prime}$-bipyridyl), $\left(\mathrm{Cr}_{7} \mathrm{Ni}\right)_{2}$-bipy in short. The magnetic coupling between the rings arises from the pyridyl groups that link $d i$ rectly-i.e. without requiring additional magnetic ions in the linker-the $\mathrm{Ni}$ ions.

Experimental characterization.-In order to magnetically characterize the single purple- $\mathrm{Cr}_{7} \mathrm{Ni}$ ring, we first measure the low-temperature specific heat $(C)$, at different applied magnetic fields $(B)$. Specific heat measurements are performed on pressed microcrystals by using the twotau method. The $C(T, B=0)$ curve shows a dominant lattice contribution at high temperature, while the magnetic term is characterized by a Schottky anomaly at around $4 \mathrm{~K}$, mainly due to the doublet-quadruplet energy difference; no evidence of a level splitting appears below $1 \mathrm{~K}$ [Fig. 1(a)]. The appearance, for $B \neq 0$, of a well defined Schottky anomaly at low temperature and its dependence on the magnetic field essentially reflect the removal of the Kramers degeneracy in the ground-state doublet. In the 


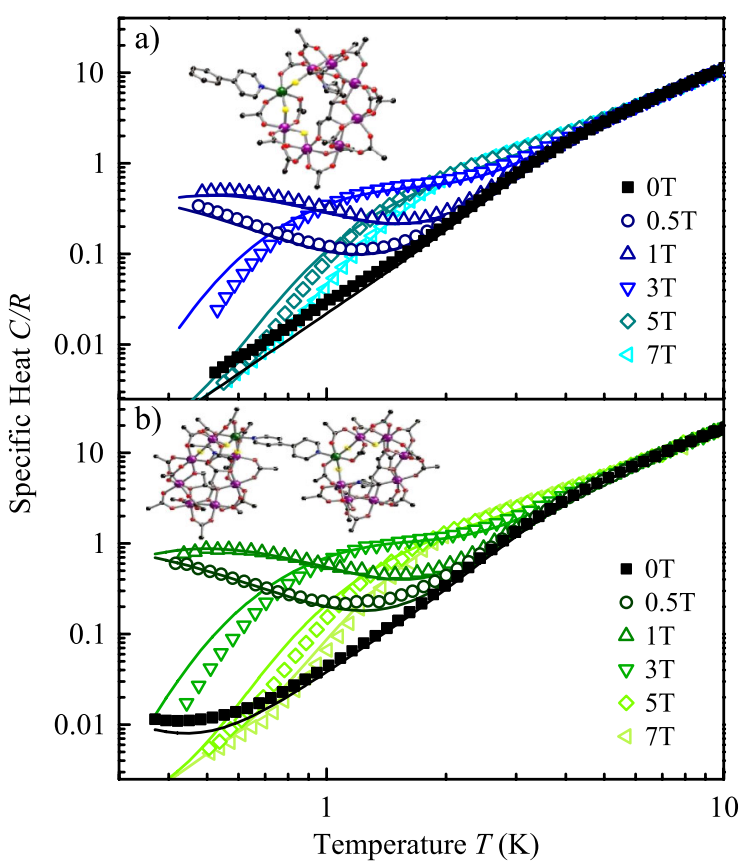

FIG. 1 (color online). Low-temperature specific heat $C$, normalized to the gas-constant $R$, of single purple- $\mathrm{Cr}_{7} \mathrm{Ni}$ rings (a) and of $\left(\mathrm{Cr}_{7} \mathrm{Ni}\right)_{2}$-bipy dimers (b). Symbols and solid lines correspond to experimental data and theoretical simulations, respectively, for different values of the applied magnetic field. Insets: structure of the single purple- $\mathrm{Cr}_{7} \mathrm{Ni}$ ring and of the dimer, as determined by $\mathrm{x}$-ray diffraction: $\mathrm{Cr}$ (purple [medium gray]), $\mathrm{Ni}$ (green [dark gray]), F (yellow [lightest gray]), O (orange [light gray]).

specific heat curve $C(T, B=0)$ of the $\left(\mathrm{Cr}_{7} \mathrm{Ni}\right)_{2}$-bipy dimer, instead, an upturn is clearly visible below $0.5 \mathrm{~K}$ [Fig. 1(b)]. This feature reveals the presence of a weak interaction between the two purple- $\mathrm{Cr}_{7} \mathrm{Ni}$ rings and allows a direct estimate of its magnitude.

Low-temperature magnetization $M$ measurements, obtained by micro-SQUID on a single crystal, allow us to further characterize the inter-ring interaction and to detect entanglement between the rings. The dc susceptibility is defined as $\chi=d M / d B$ for small values of $B$, and is measured with sweeping rates that are small enough to keep the system at the thermal equilibrium with the cryostat $\left(d B / d t=0.004 \mathrm{~T} \mathrm{~s}^{-1}\right)$. The downwards curvature of $\chi T$ below $0.2 \mathrm{~K}$ [Fig. 2(a)] demonstrates the presence of a weak antiferromagnetic coupling between the two rings. The value of $\chi$, required for experimentally demonstrating entanglement between the two rings, was normalized by using as a reference the high temperature value measured on powders. In Fig. 2(b) we report the magnetization curves $M(T, B)$ of the $\left(\mathrm{Cr}_{7} \mathrm{Ni}\right)_{2}$-bipy dimer. Here, the sweeping rate is faster $\left(d B / d t=0.280 \mathrm{~T} \mathrm{~s}^{-1}\right)$, which allows us to keep the temperature of the spin bath even lower than that of the cryostat (see Ref. [16] for a systematic study of $M(T, B)$ as a function of the sweeping rate and for comparison with other derivatives). The butterfly shape is characteristic of the phonon-bottleneck regime [17,18] and
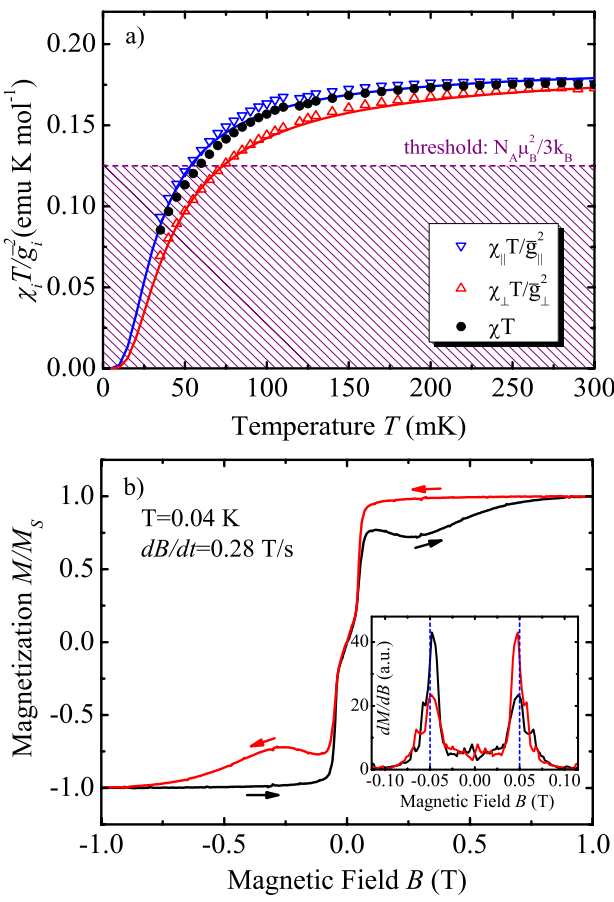

FIG. 2 (color online). (a) Temperature dependence of the measured (triangles) and simulated (solid lines) dc susceptibility components for the ring dimer. $\chi_{\perp}$ (red [medium gray]) is the component perpendicular to the largest surface of the crystal; this direction forms on average an angle of $16^{\circ}$ with the $z$ axis, perpendicular to the rings plane. $\chi_{\|}$refers to the directions parallel to the crystal plane; rotation of magnetic field within this plane does not evidence changes in the magnetic response. The average $\left(\chi_{\perp} / \bar{g}_{\perp}^{2}+2 \chi_{\|} / \bar{g}_{\|}^{2}\right) T / 3$ (circles) is compared with the threshold for a mole of dimers, $N_{A} \mu_{B}^{2} / 3 k_{B}$, in order to identify the temperature range where the two rings are entangled (shaded area). (b) Magnetization loop measured on a single crystal of $\left(\mathrm{Cr}_{7} \mathrm{Ni}\right)_{2}$-bipy at $T=40 \mathrm{mK}\left(d B / d t=0.280 \mathrm{~T} \mathrm{~s}^{-1}\right)$. Inset: $d M / d B$ vs $B$, that evidences the level crossing at $B \simeq \pm 0.05 \mathrm{~T}(\mathbf{B} \| \hat{\mathbf{x}})$.

is common to the single uncoupled ring. The curve corresponding to the $\left(\mathrm{Cr}_{7} \mathrm{Ni}\right)_{2}$-bipy dimer additionally presents feeble knees at $B= \pm 0.05 \mathrm{~T}$, which is clearly visible in the plot of $d M / d B$ vs $B$ [inset of Fig. 2(b)]. This feature, which has never been observed for uncoupled $\mathrm{Cr}_{7} \mathrm{Ni}$ rings, indicates a crossing between low and high spin states of the dimer as $B$ is swept from zero to finite values.

The above experimental results show that $\mathrm{Cr}_{7} \mathrm{Ni}$ rings can be magnetically coupled, while preserving their individual magnetic features, including the ground-state Kramers doublet. It is worth stressing that low-temperature magnetization measurements of a $\left(\mathrm{Cr}_{7} \mathrm{Ni}\right)_{2}$-dipy dimer with a different linker show a different values of the inter-ring coupling (see Ref. [16]). This further demonstrates the ability to control the coupling between $\mathrm{Cr}_{7} \mathrm{Ni}$ rings by using supramolecular chemistry.

Microscopic modeling of the dimer-Despite the complexity of these supramolecular systems, all the above experimental results can be theoretically interpreted by means of a microscopic spin-Hamiltonian approach. 
The spin Hamiltonian corresponding to the single molecular ring reads $[19,20]$ :

$$
\begin{aligned}
\mathcal{H}= & J \sum_{i=1}^{8} \mathbf{s}_{i} \cdot \mathbf{s}_{i+1}+\sum_{i=1}^{8} d_{i}\left[s_{z, i}^{2}-s_{i}\left(s_{i}+1\right) / 3\right] \\
& +\sum_{i<j=1}^{8} D_{i j}\left[2 s_{z, i} s_{z, j}-s_{x, i} s_{x, j}-s_{y, i} s_{y, j}\right] \\
& +\mu_{B} \sum_{i=1}^{8} \mathbf{B} \cdot \mathbf{g}_{i} \cdot \mathbf{s}_{i},
\end{aligned}
$$

where $z$ is the ring axis, site 8 corresponds to the $\mathrm{Ni}^{2+}(s=$ $1)$ ion and sites $1-7$ to $\mathrm{Cr}^{3+}(s=3 / 2)$. The four terms in $\mathcal{H}$ account for the isotropic exchange, the axial contributions to the crystal field and the intracluster dipole-dipole, and for the Zeeman interactions, respectively. While the values of the crystal-field parameters are assumed to coincide with those of the green- $\mathrm{Cr}_{7} \mathrm{Ni}$ compound [21,22], the exchange parameter $J / k_{B}=22.9 \mathrm{~K}$ is determined by simultaneously fitting $C(T, B)$ at different magnetic field strengths and the temperature-dependence of $\chi(T)$, measured on powders between 2 and $300 \mathrm{~K}$ (see Fig. 1 and Ref. [16]). This leads to a splitting $\Delta \simeq 19 \mathrm{~K}$ between the $S=1 / 2$ ground doublet and the first $S=3 / 2$ excited quadruplet. Analysis of the EPR data (Fig. 3) allows a precise estimate of the gyromagnetic tensor components. The single purple- $-\mathrm{Cr}_{7} \mathrm{Ni}$ ring spectrum is characteristic of a ground-state doublet, with lines sharper than those measured in green- $\mathrm{Cr}_{7} \mathrm{Ni}$; this might be due to the $F$-substitution, that reduces the hyperfine-induced decoherence [23]. Additional structures appear in the EPR spectrum of the $\left(\mathrm{Cr}_{7} \mathrm{Ni}\right)_{2}$-bipy dimer with respect to the single-ring case (Fig. 3), clearly showing interactions between the two molecular rings and mainly involving the ground doublets of the two rings. The effective values for the $g$ tensor of the ground doublet are: $\bar{g}_{x x}=\bar{g}_{y y}=1.84$ and $\bar{g}_{z z}=1.78$. These can be reproduced by assuming a slightly anisotropic Ni gyromagnetic tensor $\left(g_{x x}^{\mathrm{Ni}}=g_{y y}^{\mathrm{Ni}}=\right.$ 2.19, $g_{z z}^{\mathrm{Ni}}=2.24$ ).

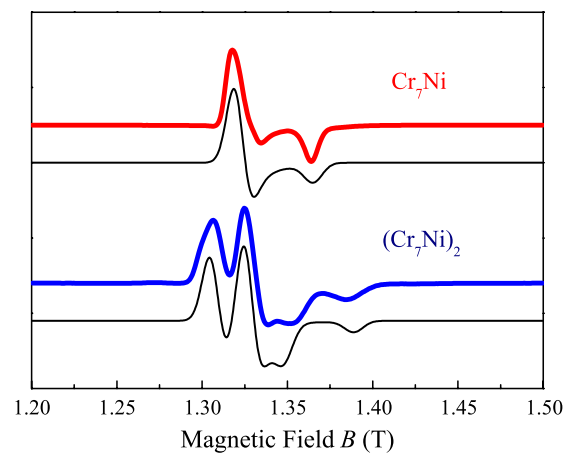

FIG. 3 (color online). Simulated $Q$-band EPR spectra of purple- $\mathrm{Cr}_{7} \mathrm{Ni}$ and $\left(\mathrm{Cr}_{7} \mathrm{Ni}\right)_{2}$-bipy dimer (solid black lines), obtained within the microscopic spin-Hamiltonian approach (see text). The experimental data (colored [gray] lines), taken from Ref. [15], have been obtained at $T=5 \mathrm{~K}$.
The microscopic intermolecular coupling between the two rings (labeled $A$ and $B$ ) in the $\left(\mathrm{Cr}_{7} \mathrm{Ni}\right)_{2}$ dimer is described by an isotropic Heisenberg term involving the $\mathrm{Ni}$ ions:

$$
\mathcal{H}_{A B}=J^{\prime} \mathbf{s}_{8}^{A} \cdot \mathbf{s}_{8}^{B} .
$$

In order to calculate the low-energy $(E)$ properties of the dimer, we first diagonalize the single-ring Hamiltonian. The coupling Hamiltonian $\mathcal{H}_{A B}$ is then represented on the product basis $\left|i_{A}, j_{B}\right\rangle$, being $\left|i_{\alpha}\right\rangle$ the single-ring eigenstates of $\mathcal{H}$. Since $J^{\prime} \ll J$, the low- $E$ eigenstates are well approximated by truncating the product basis to include only the four lowest-lying multiplets of the rings. The resulting simulations are compared with the experimental results and contrasted with the corresponding quantities obtained for single rings, in order to highlight the effect of inter-ring coupling. Specific heat [Fig. 1(b)], dc susceptibility [Fig. 2(a)] and EPR (Fig. 3) data of $\left(\mathrm{Cr}_{7} \mathrm{Ni}\right)_{2}$ are well reproduced by assuming $J^{\prime} / k_{B}=0.16 \mathrm{~K}$. The sign of $J^{\prime}$ is fixed by the low- $T$ decrease of the de susceptibility [Fig. 2(a)]. At 0.05 T our model also predicts a ground-state crossing involving one of the triplet states [Fig. 4(b)], in excellent agreement with low-temperature magnetization data [Fig. 2(b)].

Entanglement between the rings.-The quantitative agreement between our microscopic spin-Hamiltonian approach and the whole set of experimental data, based on the use of only four free parameters $\left(J, J^{\prime}, g_{x x}^{\mathrm{Ni}}=g_{y y}^{\mathrm{Ni}}\right.$, and $\left.g_{z z}^{\mathrm{Ni}}\right)$, demonstrates a detailed understanding of the intra- and inter-ring interactions and allows the derivation for the dimer of a two-qubit picture. Within such a picture, we discuss the experimental demonstration, through magnetic susceptibility, of entanglement between the two rings, and its theoretical quantification through concurrence. In fact, for $T \ll J / k_{B}$ [9], each $\mathrm{Cr}_{7} \mathrm{Ni}$ ring can be regarded as an effective $1 / 2$ spin, and the effect of intermolecular coupling can be described by projecting $\mathcal{H}^{A B}$ onto the lowenergy subspace $\mathcal{S}=\left\{\left|i_{A}, j_{B}\right\rangle\right\}$, with $i_{A}, j_{B}=1$, 2. The resulting effective Hamiltonian, expressed as a function
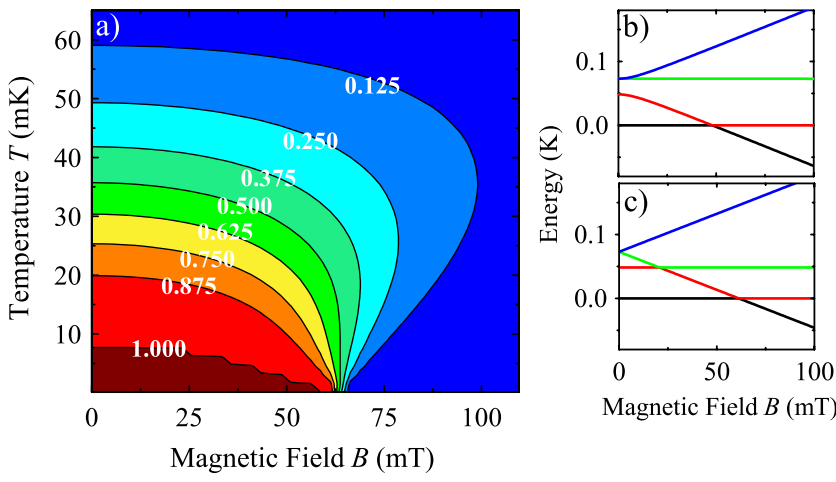

FIG. 4 (color online). (a) Concurrence of the $\left(\mathrm{Cr}_{7} \mathrm{Ni}\right)_{2}$ dimer at equilibrium at temperature $T$ in the presence of an external magnetic field $B$ (along $z$ ). Panels in the right-hand side show the low-energy spectrum of the dimer, derived from the microscopic spin Hamiltonian, for $\mathbf{B} \| \hat{\mathbf{x}}$ (b) and $\mathbf{B} \| \hat{\mathbf{z}}$ (c). 
of the total spins of the two rings, reads:

$$
\mathcal{H}_{0}^{\mathrm{eff}}=J_{A B} \mathbf{S}_{A} \cdot \mathbf{S}_{B}+D_{A B}\left(2 S_{z}^{A} S_{z}^{B}-S_{x}^{A} S_{x}^{B}-S_{y}^{A} S_{y}^{B}\right),
$$

where $J_{A B} \simeq 0.4 J^{\prime}$ and $D_{A B} \simeq 0.1 J^{\prime}$. Here, the second contribution is due to anisotropic crystal-field and intramolecular dipolar interactions. In the presence of a magnetic field, the Hamiltonian of the coupled effective spins $S_{A}=$ $S_{B}=1 / 2$ thus reads $\mathcal{H}^{\text {eff }}=\mathcal{H}_{0}^{\text {eff }}+\mathcal{H}_{1}^{\text {eff }}$ with $\mathcal{H}_{1}^{\text {eff }}=$ $\mu_{B} \sum_{\alpha=A, B} \mathbf{B} \cdot \overline{\mathbf{g}} \cdot \mathbf{S}_{\alpha}$.

For a system of exchange-coupled spins, the absolute value of the magnetic susceptibility, averaged over three orthogonal directions, represents an entanglement witness [24]: if $\sum_{\beta} \chi_{\beta} T / \bar{g}_{\beta}^{2}<N_{A} \mu_{B}^{2} / k_{B}$, then the density matrix $\rho$ of the two effective spins $\mathbf{S}_{\alpha}$ is nonfactorizable; here, $\beta=$ $x^{\prime}, y^{\prime}, z^{\prime}$ are the three axes and the threshold refers to an Avogadro number of dimers. As shown in Fig. 2, for $T \lesssim$ $50 \mathrm{mK}$, the above inequality is fulfilled, thus providing a direct experimental evidence of entanglement between the two $\mathrm{Cr}_{7} \mathrm{Ni}$ rings. In the present case, the coupling between the effective spins $\mathbf{S}_{\alpha}$ includes an anisotropic contribution $\left(D_{A B} \neq 0\right)$, due to which the condition $\left[\mathcal{H}_{0}^{\text {eff }}, \mathcal{H}_{1}^{\text {eff }}\right]=0$ is only fulfilled for $\mathbf{B} \| \hat{\mathbf{z}}$. This might in principle result in a difference between the magnetic susceptibility and the entanglement witness [23]. However, for values of $D_{A B} / J_{A B}=0.25$ (or smaller), such difference corresponds to less than $2 \%$ of the threshold value (see Ref. [16]).

A quantitative, though indirect, estimate of entanglement is provided by the concurrence $(\mathcal{C})$ [1], whose value ranges from 0 for factorizable states to 1 for maximally entangled ones. The equilibrium density matrix of the dimer $\rho_{\mathrm{eq}}^{A B}$, in the case $\mathbf{B} \| \hat{\mathbf{z}}$, is a statistical mixture of the eigenstates $|S, M\rangle$, each weighted by its Boltzmann factor $P_{S, M}$. The resulting expression of the concurrence is $\mathcal{C}\left(\rho_{\mathrm{eq}}^{A B}\right)=\max \left\{\left|P_{1,0}-P_{0,0}\right|-2\left(P_{1,1} P_{1,-1}\right)^{1 / 2}, 0\right\}, \quad$ if $\max \left\{P_{1,0}, P_{0,0}\right\}>\left(P_{1,1} P_{1,-1}\right)^{1 / 2}$, and $\mathcal{C}\left(\rho_{\text {eq }}^{A B}\right)=0$ otherwise. In the range where the concurrence is finite, its dependence on $T$ and on the physical parameters in $\mathcal{H}^{\text {eff }}$ is given by the following expression [Fig. 4(a)]:

$$
\mathcal{C}\left(\rho_{\mathrm{eq}}^{A B}\right)=\frac{1-e^{-\left(J_{A B} / k_{B} T\right)}\left[e^{\left(D_{A B} / k_{B} T\right)}+2 e^{-\left(D_{A B} / 2 k_{B} T\right)}\right]}{1+e^{-\left(J_{A B} / k_{B} T\right)}\left[e^{\left(D_{A B} / k_{B} T\right)}+2 e^{-\left(D_{A B} / 2 k_{B} T\right)} \cosh \left(\bar{g}_{z z} \mu_{B} B / k_{B} T\right)\right]} .
$$

For $B=0$, the dimer is maximally (hardly) entangled if the temperature is much smaller than (comparable to) the singlet-triplet splitting $E_{1,0}-E_{0,0}=J_{A B}-D_{A B}$. The transition from entangled to factorizable states corresponds to $T \simeq 50 \mathrm{mK}$, in agreement with that estimated through the entanglement witness. At vanishing temperature, the concurrence undergoes an abrupt transition from $\mathcal{C}=1$ to $\mathcal{C}=0$ as the value of the magnetic field exceeds $B_{c}=$ $\left(J_{A B}+D_{A B} / 2\right) / \mu_{B} \bar{g}_{z z}$, where, a crossing takes place between $|S=0, M=0\rangle$ and $|S=1, M=1\rangle$ [Fig. 4(c)]. Altogether, the two rings are entangled in the temperature range $\bar{g}_{z z} \mu_{B}\left(B-B_{c}\right) \lesssim k_{B} T \lesssim J_{A B}-D_{A B}$, that includes experimental conditions reported in the manuscript.

In summary, we have characterized in detail a dimer of magnetically coupled $\mathrm{Cr}_{7} \mathrm{Ni}$ rings and provided direct experimental evidence of entanglement between their spins by using magnetic susceptibility as entanglement witness. Besides, based on a microscopic modeling of the dimer, we have quantified the ring-ring entanglement by calculating the concurrence, and obtained finite values for an experimentally accessible range of physical parameters.

This work has been carried out within the framework of the EU Network of Excellence "MAGMANet" Contract No. 515767 and supported by FP7-ICT FET Open "MolSpinQIP" project, Contract No. 211284 and by the EPSRC (UK).

[1] L. Amico et al., Rev. Mod. Phys. 80, 517 (2008).

[2] S. Ghosh et al., Nature (London) 48, 425 (2003).
[3] M. Nielsen et al., Quantum Computation and Quantum Information (Cambridge University Press, Cambridge, England, 2000).

[4] D. Gatteschi et al., Molecular Nanomagnets (Oxford University Press, Oxford, 2007).

[5] W. Wernsdorfer et al., Nature (London) 416, 406 (2002).

[6] S. Hill et al., Science 302, 1015 (2003).

[7] L. Bogani and W. Wernsdorfer, Nature Mater. 7, 179 (2008).

[8] M. N. Leuenberger et al., Nature (London) 410, 789 (2001); F. Meier, J. Levy, and D. Loss, Phys. Rev. Lett. 90, 047901 (2003).

[9] F. Troiani et al., Phys. Rev. Lett. 94, 207208 (2005).

[10] S. Carretta et al., Phys. Rev. B 76, 024408 (2007).

[11] F. Troiani et al., Phys. Rev. Lett. 94, 190501 (2005).

[12] A. Ardavan et al., Phys. Rev. Lett. 98, 057201 (2007).

[13] V. Corradini et al., Phys. Rev. B 79, 144419 (2009).

[14] G. A. Timco et al., Nature Nanotech. 4, 173 (2009).

[15] G. Timco et al., Angew. Chem., Int. Ed. 47, 9681 (2008).

[16] See supplementary material at http://link.aps.org/ supplemental/10.1103/PhysRevLett.104.037203 for further details on the fitting procedure, on the use of $\chi$ as entanglement witness, and on a dimer with a different linker.

[17] I. Chiorescu et al., Phys. Rev. Lett. 84, 3454 (2000).

[18] W. Wernsdorfer et al., Phys. Rev. B 72, 060409(R) (2005).

[19] S. Carretta et al., Phys. Rev. B 67, 094405 (2003).

[20] R. Caciuffo et al., Phys. Rev. B 71, 174407 (2005).

[21] These are: $d_{\mathrm{Cr}}=-0.35 \mathrm{~K}$ and $d_{\mathrm{Ni}}=-4 \mathrm{~K}$. The dipolar coefficients $D_{i j}$ have been calculated in the point-dipole approximation. Finally, the gyromagnetic tensor components of the $\mathrm{Cr}$ ions are: $g_{x x}^{1-7}=g_{y y}^{1-7}=g_{z z}^{1-7}=1.98$.

[22] S. Carretta et al., Phys. Rev. B 72, 060403(R) (2005).

[23] F. Troiani, V. Bellini, and M. Affronte, Phys. Rev. B 77, 054428 (2008).

[24] M. Wieśniak et al., New J. Phys. 7, 258 (2005). 